\title{
EFFECT OF AUTO-FOCUSING OF THE ELECTRON BEAM IN THE RELATIVISTIC VACUUM DIODE
}

\author{
S. Adamenko, E. Bulyak *, V. Stratienko, N. Tolmachev, Ukraine
}

\begin{abstract}
A hypothesis of the beam auto-focusing in the relativistic vacuum diode with a needle anode is proposed. A physical model of the effect is elaborated. As is shown, auto -focusing of high current beam can be caused by ions emitting from the anode. Initial (pre focusing) stage of focusing is provided with ionization of the gas molecules absorbed in the anode surface. Then (at quasi-steady stage), the focusing is driven by the metal ions being evaporated from the anode heated by the beam. Simulation of the process shown the duration of initial pre focusing stage would be within $0.1-0.2 \mathrm{nsec}$.
\end{abstract}

\section{INTRODUCTION}

Experiments on electrical discharge in the relativistic vacuum diode with a needle anode yielded strong focusing of the electron beam in the anode. Such a device has some micrometers radius of the anode and a few millimeters the cathode-anode gap. After applying $1 \mathrm{MV}$ pulse of nanosecond duration, a tiny hole along the anode axis was appeared [1]. It is obvious that beam focusing into a small fraction of the anode surface for a relatively long time could be possible if a feedback presents. The paper aimed at physical ground of the phenomenon called the beam self-focusing.

\section{MODEL}

It is physically reasonable that focusing of the moderately relativistic beam into a small crossover requires a shortfocus lens to be placed at the anode. This lens might be provided with axially symmetrical cloud of positively charged ions.

Presence the ions themselves is not sufficient for the entire current pulse to be focused. The matter is that the time interval for which an electron crossed the cathode-anode gap sufficiently (by 3-4 orders of magnitude) shorter than the pulse duration.

Therefore, during a pulse the beam parameters such as current, energy of electrons, density of ions undergo variations. Due to these variations the focus spot is deviated. Thus, explanation of the self-focusing is required presence of the feed-back - the ion density must be bounded to the spot size.

\footnotetext{
*Email: bulyak@kipt.kharkov.ua
}

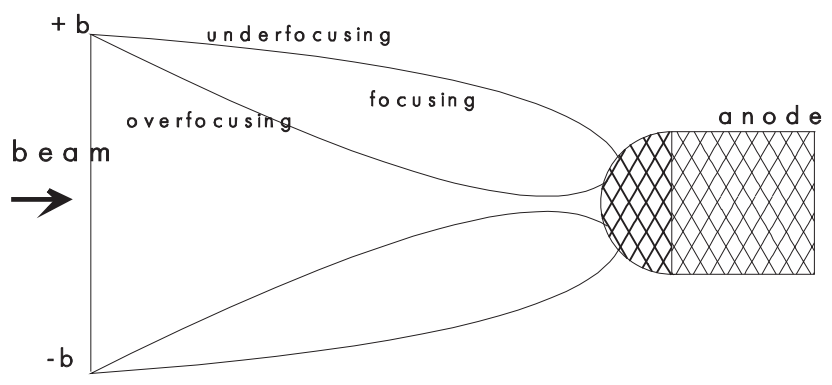

Figure 1: Anode half of the relativistic diode

Let us consider the anode surface being covered with a thin layer of neutral atoms (molecules). Also, suppose the density of the layer dependent on the density of the beam hitting upon this layer. The equation of the neutrals balance can be read as

$$
\frac{\mathrm{d} \nu_{0}}{\mathrm{~d} t}=-\lambda \nu_{0}+\kappa \dot{\nu_{b}}(t)
$$

where $\nu_{0}$ is the surface density of the 'vapor'; $\dot{\nu_{b}}=n_{b}(t) \beta c$ is the density of electron flux onto the anode; $\beta c$ is the velocity of electrons; $\lambda$ is the coefficient of 'condensation'; $\kappa$ is 'the conversion factor' (number of neutrals produced by an electron hitting the anode).

A solution to (1) with the initial condition $\nu_{0}=\nu(0)$ at $t=0$ has the form:

$$
\begin{aligned}
\nu_{0}(t)= & \exp (-\lambda t)\left[\nu^{(0)}+\beta c \frac{\kappa}{\lambda}\right. \\
& \left.\times \int_{0}^{t \lambda} n_{b}(\tau / \lambda) \exp (\tau) \mathrm{d} \tau\right], \\
& \lim _{t \rightarrow \infty} \nu_{0}(t)=n_{b} \beta c \frac{\kappa}{\lambda} .
\end{aligned}
$$

The ions produced from ionization of the neutrals in the layer are accelerated in the near-to-anode space up to velocity $V_{i}$. Density of the ions beyond the accelerating space is satisfied to the discontinuity equation:

$$
\frac{\partial n_{i}}{\partial t}+\operatorname{div}\left(\vec{V}_{i} n_{i}\right)=0
$$

For the case of spherical geometry we have:

$$
\begin{aligned}
n_{i}(r, t)= & \left(\frac{r_{a}}{r}\right)^{2} n_{i}\left(r_{a}, t^{\prime}\right), \\
& t^{\prime} \equiv t-\frac{r-r_{a}}{V_{i}} .
\end{aligned}
$$


where

$$
n_{i}\left(r_{a}, t\right)=\frac{n_{b}(t) \beta c \sigma_{i}}{V_{i}} n_{b} \beta c \frac{\kappa}{\lambda},
$$

$\sigma_{i}$ is the ionization cross section.

The ion cloud around the anode produces the electrostatic potential

$$
\begin{aligned}
\Phi(r, t)= & \Phi_{a}+\frac{r_{c}\left(r-r_{a}\right)}{r\left(r_{c}-r_{a}\right)} \\
& {\left[\Phi_{c}-\Phi_{a}+\Psi\left(r_{c}, t\right)\right]-\Psi(r, t) ; } \\
& \Psi(r, t) \equiv \frac{e r_{a}^{2}}{\epsilon_{0}} \int_{r a}^{r} \frac{\mathrm{d} r^{\prime}}{r^{\prime 2}} \\
& \times \int_{r a}^{r^{\prime}} n_{i}\left(r_{a}, t-\frac{r^{\prime \prime}-r_{a}}{V_{i}}\right) \mathrm{d} r^{\prime \prime} .
\end{aligned}
$$

Here $e$ is the electron charge. $\Phi_{a, c}$ is the voltage of the anode and cathode, respectively.

For the time-independent ion cloud density in the nearto-anode layer, $n_{i}\left(r_{a}, t\right)=$ const $=n_{i}\left(r_{a}\right)$,

$$
\Psi(r)=\frac{e n_{i}\left(r_{a}\right) r_{a}^{2}}{\epsilon_{0}}\left[\ln \frac{r}{r_{a}}-\frac{r-r_{a}}{r}\right]
$$

Let consider the cylindrical parallel electron with the radius $b$ and density $n_{b}(\rho)$ enters the system at the velocity $\beta c$. Being focused by the positive ions that left the spherical cathode with the velocity $V_{i}$, the $\eta$ fraction of the beam hits the anode and produces new ions from the near-toanode neutrals (density of neutrals is $\nu_{0}$ ). Also consider the steady-state and neglect the self-beam space charge that can change the only quantitative (not qualitative) features of the process [2], we come to the potential of the form:

$$
\begin{array}{r}
\Phi(r)=\text { const }+\Psi(r)= \\
\text { const }+\frac{e n_{i}\left(r_{a}\right) r_{a}^{2}}{\epsilon_{0}}\left(\ln \frac{r}{r_{a}}-\frac{r-r_{a}}{r}\right) \approx \\
\text { const }+\frac{e n_{i}\left(r_{a}\right) r_{a}^{2}}{\epsilon_{0}} \ln \frac{r}{r_{a}},
\end{array}
$$

where $r_{0}$ is the classical electron radius.

In the cylindrical geometry $\left(r^{2}=\rho^{2}+\left(z-z_{s}\right)^{2}\right)$, an equation describing the beam envelope reads as:

$$
\begin{aligned}
y^{\prime \prime}+k \frac{y}{x^{2}} & =0 ; \\
k & \equiv r_{0} \frac{4 \pi n_{i}\left(r_{a}\right) r_{a}^{2}}{\gamma \beta^{2}} ; \\
y \equiv \rho / r_{a} ; x \equiv z / r_{a} . &
\end{aligned}
$$

A solution to this equation depends on the density of ions $n_{i}^{l}$

$$
n_{i}^{*}=\frac{\gamma^{2}+1}{16 \pi r_{0} r_{a} \gamma}
$$

If $n_{i}\left(r_{a}\right) \gg n_{i}^{*}$, then we have

$$
\begin{gathered}
\rho(z)=\rho_{0} \sqrt{\frac{z_{s}-z}{z_{s}}} \cos \left(s \ln \frac{z_{s}-z}{z_{s}}\right) ; \\
s^{2}=\frac{4 \pi r_{0} r_{a}^{2} \gamma n_{i}\left(r_{a}\right)}{\gamma^{2}-1}=\mu I_{a}
\end{gathered}
$$

As it yields from (11), at the specific density of ions $n_{i}^{\mathrm{f}}$ for the given system geometry, the whole beam is focused into the anode $(\eta=1)$ :

$$
n_{i}^{\mathrm{f}}=\frac{\pi z_{s}^{2}\left(\gamma^{2}+1\right)}{16 r_{a}^{4} \gamma r_{0}} .
$$

The beam current providing the spot not exceeding the anode transverse dimension, spans the interval:

$$
I_{ \pm}=\left[\frac{z_{s}}{\mu r_{a}}\left(\frac{\pi}{2} \mp \frac{r_{a}}{b}\left(1+\frac{r_{a}}{2 z_{s}}\right)\right)\right]^{2} .
$$

the magnitude of current is proportional to $\left(z_{s} / r_{a}\right)^{2}$, while the relative width

$$
\frac{\Delta I}{I}=\frac{4 r_{a}}{\pi b} .
$$

In other words, for bigger demultiplication (ratio of the initial beam radius to that at the anode) the focusing takes place within the narrower interval of the beam current.

\section{SIMULATION OF THE TRANSITING STAGE}

The consideration presented above indicates that under the certain conditions (presence of neutral atoms in the anode surface) the steady beam focusing into the anode can be achieved. Another necessary condition is 'attraction' of the beam to the focused state: the system shall transit itself into the stable focused state.

The computer code has been written to simulate evolution in time of the envelope of initially parallel beam. The code inherits the potential (7) and the source of neutrals (2). The species of neutral atoms can cover either all the anode semisphere or a near-to-axis circle. Variations in time in the ion density are self-consistent, according to (6).

Results of simulation of the transition regime show out that capturing the beam into the focused state requires the neutral atoms of constant (in time) density or the 'evaporation-condensation' eqiullibrium to be presented in the anode surface. Latter produces capturing in less time, besides the focal area being smaller. In any case for the transition into focused state, it is necessary the neutrals to cover the anode surface. 'Condensing' means that these neutrals decrease in number due to ionization.

The sample of the beam radius evolution is presented in Fig 2. Also it was found duration of the transition depends on the density of absorbed neutrals. 


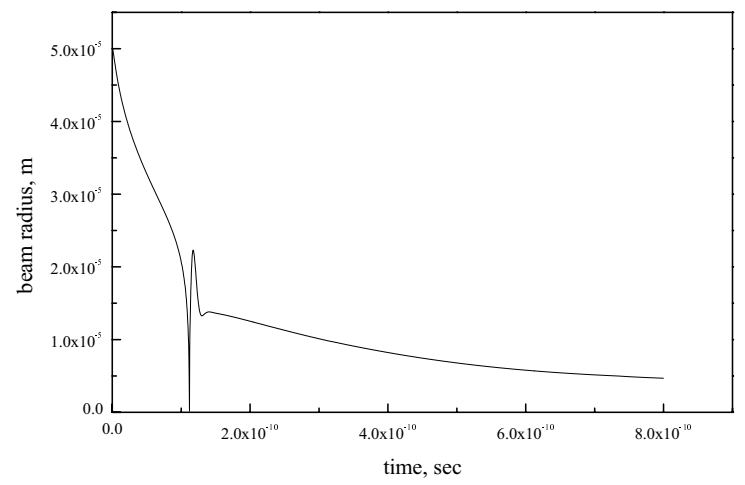

Figure 2: The beam radius evolution

\section{SUMMARY}

The study proves the hypothesis of the beam self-focusing with ions produced from the anode 'vapor'. At first warming-up stage, the neutrals absorbed in the anode are involved. After, the self-stabilizing mechanism of focusing due to evaporation of the neutrals from the anode is driven.

The width of the interval of current where the entire beam comes into the anode head surface, is proportional to ratio of the anode radius to the initial radius of the beam. The beam space charge will shift the focused beam current to larger values. Also it was shown that the only overfocused state can be equillibrium. The time required to reach the steady state can be less than a nanosecond. The results of the numerical simulation are in qualitative agreement with the experimental data: the self-focusing of the beam with energy of particles $0.5-1 \mathrm{MeV}$ and current of a few kA can be produced at the distance of a few millimeters for less than a nanosecond.

\section{REFERENCES}

[1] V.A. Stratienko, S.V. Adamenko, A.N. Dovbnya, et al. "Generation and Application of the Dense Secodary Emitting Beams," in: VANT, ser. YaFI, (1997), No 2,3 (29,30), p 296

[2] E. Bulyak, "Ion clearing methods for the electron storage rings," in Proc of EPAC-96 (Barcelon, Spain), 1996, p. 1048 